\title{
One-dimensional dynamics in locally heated liquid layers
}

\author{
J. Burguete*, D. Maza, H.L. Mancini \\ Departamento de Física y Matemática Aplicada, Universidad de Navarra, Irunlarrea $s /$, \\ Apdo. 177, E-31080 Pamplona, Spain
}

Received 6 October 2001; received in revised form 19 February 2002; accepted 21 May 2002

\begin{abstract}
Recent results on one-dimensional patterns in locally heated experiments are presented. A fluid layer is heated locally by a nearly one-dimensional heater, and subjected to both horizontal and vertical temperature gradients. Depending on the fluid depth and on the temperature difference established across the layer different convective regimes appear. When a very small temperature gradient is applied a basic convective state appears. It consists of two big rolls parallel to the heater and filling the convective cell. A primary instability in the homogeneous basic flow gives rise to a one-dimensional cellular stationary pattern. For higher values of the control parameters, time-dependent patterns appear through a secondary instability. Various regimes are analyzed: oscillations, traveling waves and alternating patterns. The hydrodynamic characteristics of these patterns are provided. Local temperature measurements allows to describe the physical mechanisms responsible for the instabilities. The similarities and discrepancies of the experimental data with some theoretical models are provided.
\end{abstract}

(C) 2002 Elsevier Science B.V. All rights reserved.

PACS: 47.35.+i; 47.20.-k; 05.45.-a; 47.27.Te

Keywords: Nonlinear waves; One-dimensional patterns; One-dimensional dynamics

\section{Introduction}

The analysis of one-dimensional patterns has been widely studied in recent years. Although these systems are, a priori, easier to describe than the three- or even two-dimensional case, there are a lot of questions that remain to be answered.

In the typical scenario, an homogeneous state destabilizes to a cellular one-dimensional pattern, that can be either time-dependent or stationary. When the bifurcation leads to a time-dependent pattern, the dynamics of the large scale pattern amplitude is very well

\footnotetext{
* Corresponding author. Tel.: +34-948-425-600x6383; fax: +34-948-425-649.

E-mail address: javier@fisica.unav.es (J. Burguete).
}

understood. This kind of behavior can be found in experiments of lateral heating in thin fluid layers [1,2], hot-wire convection [3], von Kármán street flows [4], nematic liquid crystals [5], Taylor-Dean instability [7] or turbulent spirals on Taylor-Couette flow [6].

The dynamics in these systems has been understood with relative success using one-dimensional models. In particular, a complex Ginzburg-Landau model is able to reproduce a grate number of the experimental results (see for example [8,9] and references therein).

However, when the first bifurcation leads to a stationary pattern and a secondary bifurcation produces time-dependent patterns, the dynamics is poorly understood. There are a lot of experiments with a very rich phenomenology for which a whole description is not available. Experimental systems with such 
dynamics are Taylor-Couette [10], laterally heated fluid layers (for high fluid depths) [2], directional solidification [11-15], directed fingering (printer instability) [16-18], laser spectra [19], Rayleigh-Bénard [20-23] and Rayleigh-Taylor [24-28] instabilities.

Using symmetry arguments Coullet and Iooss [29] made a classification of the different kinds of secondary bifurcations that appear over one-dimensional cellular patterns. In 1991, Goldstein et al. proposed a general frame to explain the drifting domains observed inside stationary patterns in experimental systems. This theory was based in the symmetry breaking process when the secondary bifurcation takes place.

Recently, Gil [31,32] presented an extension of previous analysis in secondary instabilities that produce time-dependent patterns with an spatial period twice the stationary one. His theory explains some experimental results observed in Rayleigh-Taylor and in other systems, and predicts some results that have not been observed yet.

In this paper we present experimental results on a fluid thin layer locally heated by a nearly one-dimensional heater. The basic homogeneous state, a pair of convective rolls oriented along the heater, destabilizes in a one-dimensional cellular stationary pattern. When the control parameters are further increased, this structure destabilizes and a new secondary instability appears, giving rise to three different time-dependent patterns.

Experiments where fluid layers are locally heated has been studied in other configurations. The heating is provided by means of resistive wires placed under the surface $[3,33]$ or by a laser beam $[34,35]$. Nevertheless, the time-dependent patterns of these systems are produced after a primary bifurcation.

In previous works, we have shown the effect of gradients of the control parameter on the dynamics of the stationary pattern itself [36-38]. In this work we present the results concerning the secondary bifurcation and the characterization of the time-dependent patterns.

In Section 2, we present the experimental setup, and the results are introduced in Section 3. In Section 4, we compare with other experiments and models, and in Section 5 we resume briefly our conclusions.

\section{Experimental setup}

We consider an horizontal fluid layer of depth $d$ ( $z$-coordinate) in a container of length $l_{y}$ ( $y$-coordinate) and width $l_{x}$ ( $x$-coordinate) contained in a vessel with a rigid bottom plate and an upper surface open to the atmosphere (see Fig. 1). A heater is located in the middle of the bottom plate at $x=0$, along the $y$-direction. The width of this heater $(1 \mathrm{~mm})$ is smaller than the fluid depth, and compared to the container width. The bottom plate is made of two first surface mirrors, one at each side of the heater, to allow optical techniques. We have worked with two different containers:

- The small container (SC) is $l_{y}=25 \mathrm{~cm}$ long and $l_{x}=4 \mathrm{~cm}$ wide. The heater temperature $T_{+}$is controlled with a thermostabilized water circulation that ensures a temperature stability of $0.05 \mathrm{~K}$. The fluid layer is open to the atmosphere $\left(T_{\text {atmosph }} \simeq 25^{\circ} \mathrm{C}\right)$, and the lateral wall temperatures are not controlled. Two experimental parameters can be varied: $d$ and $\Delta T_{v}=T_{+}-T_{\mathrm{atmosph}}$.

- The big container (BC) is $l_{y}=45 \mathrm{~cm}$ long and $l_{x}=$ $6 \mathrm{~cm}$ wide. In this case the wall temperature $T_{-}$can be controlled with a secondary water circulation. A third parameter appears, the lateral temperature difference $\Delta T_{h}=T_{+}-T_{-}$.

In both cases (BC and $\mathrm{SC}$ ) the aspect ratio $\Gamma_{y}=$ $l_{y} / d$ is large enough to assume that the system is not affected by the lateral ends ( $y$-direction). This point will be verified below.

The fluid used is a $5 \mathrm{cSt}$ Rhône Poulenc silicon oil (Table 1). Although the system is open to the atmosphere, the evaporation being nearly negligible does not affect the dynamics: less than $0.05 \mathrm{~mm}$ each day. The fluid depth is determined by means of a micrometric screw with an accuracy of $50 \mu \mathrm{m}$. For the depths considered in this experiment $(1-6 \mathrm{~mm})$, the value of the vertical $\Delta T$ for which convection threshold is traversed is less than $1 \mathrm{~K}$.

We characterize the flow and the dynamics by shadowgraphy associated to image processing and by means of temperature measurements. A He-Ne laser beam is expanded and projected over the container in the $z$-direction. This beam crosses the fluid layer, 


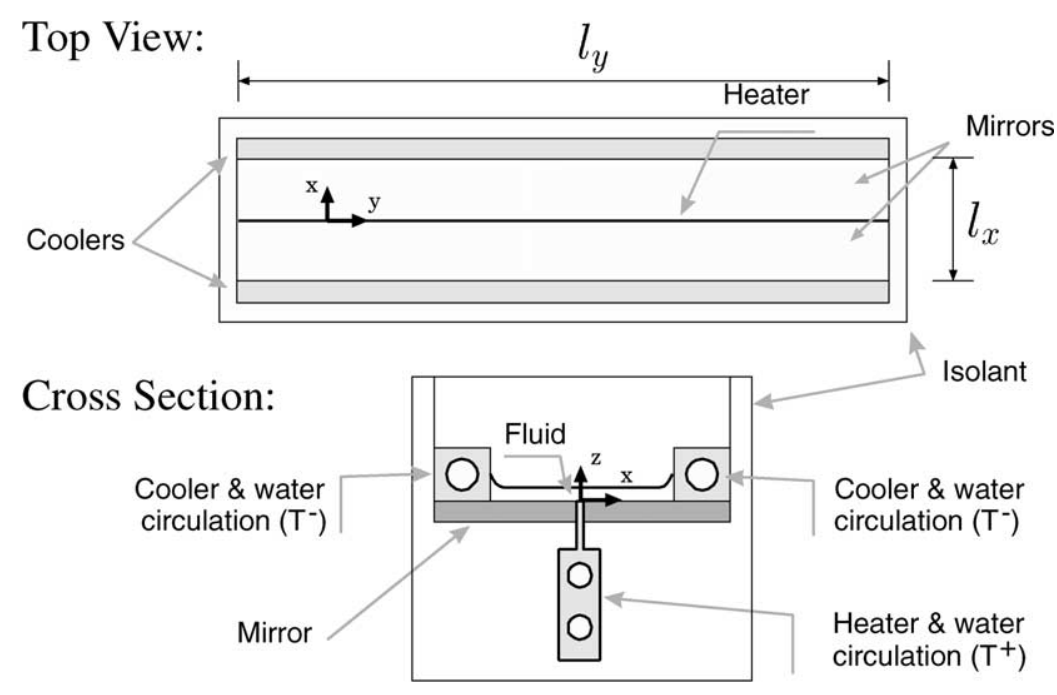

Fig. 1. Experimental setup. Sketch of the experimental cells: top view (top) and cross-section (bottom). The origin of coordinates is placed at the mirror plates in the vertical direction $z$, and at the heater in the $x$-direction. A divergent expanded laser beam crosses vertically the cell, and is projected over a screen.

reflects on the mirrors, and is projected back into a screen. Because of the largest cell size $(45 \mathrm{~cm})$, it was not practical for us to obtain a parallel beam using lenses. Instead, we have worked with an slightly divergent light beam (about $0.06 \mathrm{rad}$ ). Once screen image is recorded with a CCD camera, it is digitalized and processed in a computer, obtaining both two-dimensional photographs and spatio-temporal diagrams. Space and time Fourier transforms and complex demodulation techniques are used to extract the spatio-temporal behavior of the patterns (see details in [8] and references therein). We have determined in this way the hydrodynamic characteristics of the patterns. The divergence in the laser beam is taken into account when determining the hydrodynamic characteristics.

Two different techniques have been used to observe the thermal behavior of the fluid. First we measure the local temperature using a $0.5 \mathrm{~mm}$ diameter K-thermocouple having a characteristic response time of about $0.3 \mathrm{~s}$ when immersed in silicone oil. If the thermocouple is placed over the surface at any point near the center of the channel, the dynamics of the pattern changes drastically (for example, it fixes the secondary cell boundaries). The only non-perturbative position for the thermocouple is to place it near the lateral walls. We have therefore used this method to record the thermal oscillations only near the lateral walls.

Second, we use an optical technique to measure the temperature gradients. A laser beam entering perpendicular to the free surface and reflected at the bottom is deflected because of the local variations of the index of refraction, which depends on the temperature [39]. By measuring the oscillations of this laser beam, the ratio between the gradient along the heater and the gradient across the cell can be estimated. Moreover, from the reflection at the free surface we notice that traveling waves induce surface deflections which are less than $1 \mu \mathrm{m}$ for a depth of $3 \mathrm{~mm}$.

Table 1

Physical properties of Rhône Poulenc silicone oil $5 \mathrm{cSt}$ at $20^{\circ} \mathrm{C}$

\begin{tabular}{llllll}
\hline$\nu\left(\mathrm{m}^{2} / \mathrm{s}\right)$ & $\rho\left(\mathrm{kg} / \mathrm{m}^{3}\right)$ & $\alpha\left(\mathrm{K}^{-1}\right)$ & $\kappa\left(\mathrm{m}^{2} / \mathrm{s}\right)$ & $\sigma(\mathrm{N} / \mathrm{m})$ & $\gamma=-\partial \sigma / \partial T(\mathrm{~N} / \mathrm{mK})$ \\
\hline $5 \times 10^{-6}$ & 910 & $1.05 \times 10^{-3}$ & $6.68 \times 10^{-8}$ & $1.97 \times 10^{-2}$ & $8 \times 10^{-5}$
\end{tabular}




\section{Results}

As described in the previous works [36-38] a small $\Delta T$ between the heater and the ambient temperature is sufficient to give rise to a convective circulation across the convective cell. The fluid upon the heater becomes unstable and the Marangoni effect entrains the uprising flow, leading to a flow from the center to the lateral walls (perpendicular to the heater) where the flow descends. This forms the primary convective pair of cells, which fills the entire container and has a translational symmetry along the heater. This basic or primary flow persists in successive bifurcations.
Over this basic flow, successive instabilities appear depending on $\Delta T$ and $d$. To clarify the main situations we present the stability diagram in Fig. 2 for the small cell (top) and the effect of $\Delta T_{h}$ on the thresholds on the big cell (bottom).

Depending on the values of $d$ and $\Delta T_{v, h}$ two kinds of spatio-temporal patterns can be distinguished: stationary patterns (zone II) and time-dependent patterns (zone III). Inside these zones, various regions can be differentiated: in zone II we find one- or two-dimensional patterns, while in zone III, we find oscillations, traveling waves or alternating patterns.

The threshold values of the control parameters are different on both cells. As it can be seen in Fig. 2,

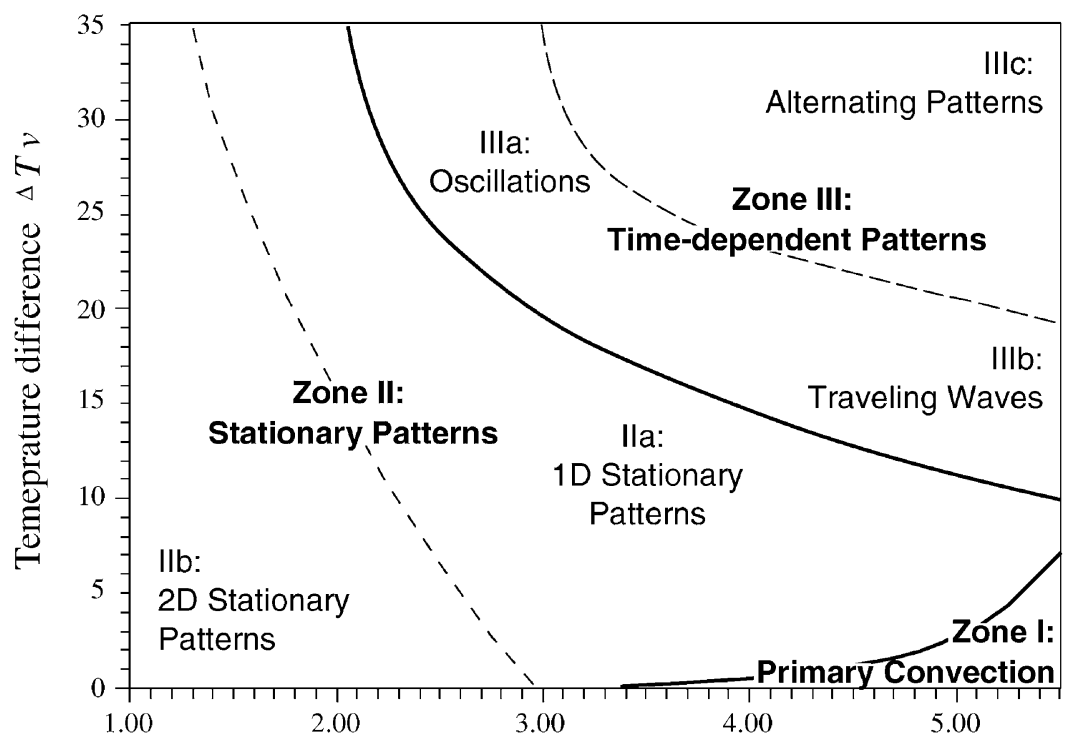

(a)

Fluid layer depth $d(\mathrm{~mm})$

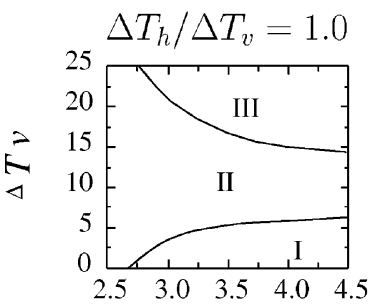

(b)
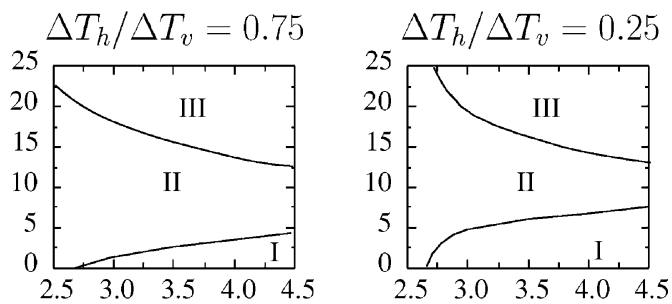

Fluid layer depth $d(\mathrm{~mm})$

Fig. 2. Stability diagram as a function of the experimental parameters $d$ and $\Delta T$. The $(d, \Delta T)$ space is divided into three main zones: basic flow, stationary and time-dependent patterns. In the last two zones, five regions can be distinguished: region IIa, stationary 1D pattern; region IIb, stationary two-dimensional pattern; region IIIa, oscillatory pattern; region IIIb, traveling waves; region IIIc, alternating pattern. Specific characteristics of each pattern are explained in the text. 
a change in $\Delta T_{h}$ moves the thresholds but does not change substantially the dynamics. The minima values of the threshold $\Delta T_{v}$ are obtained for $\Delta T_{h} \simeq$ $0.75 \Delta T_{v}$. Nevertheless, in both SC and BC cells, threshold values of $d$ and $\Delta T_{v}$ are similar. In the following, we will not distinguish between both cells unless they display significant differences.

\subsection{Stationary patterns}

Beyond a critical threshold $\Delta T_{v}^{1}$, a pattern with a well-defined wavevector parallel to the heater and a wavelength $\lambda \simeq 2 d$ appears. In this work, we focus on the analysis of the pattern near the heater. This pattern manifest as perturbations of the basic rolls as bright lines associated to the colder point of the circulation (see Fig. 3). The intensity of a line of pixels near the
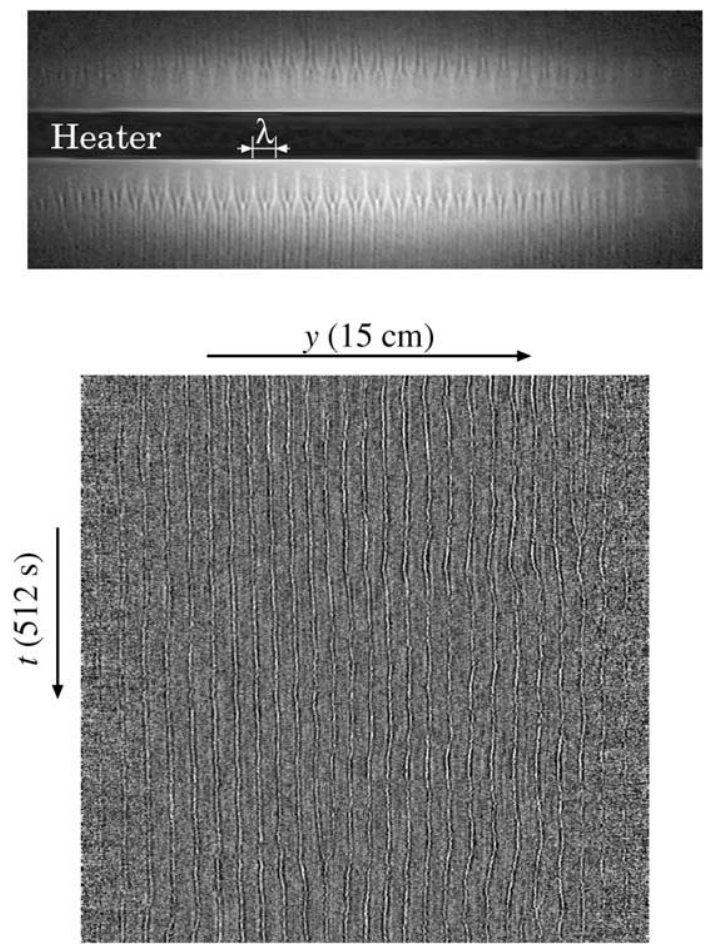

Fig. 3. (a) Shadowgraphy of the stationary pattern. The darker central part along the horizontal ( $y$-axis) corresponds to the heater, that does no reflect light, magnified by thermal lenses in the $x$-direction (in the vertical). (b) Spatio-temporal diagram of a stationary pattern for $d=2.5 \mathrm{~mm}$ and $\Delta T_{v}=20 \mathrm{~K}$. Time increases downwards. heater is recorded and stacked in the time direction and the corresponding spatio-temporal patterns are analyzed (Fig. 3, bottom).

Aluminum powder has been used for flow visualization, concluding that the original basic rolls has been broken in a cellular pattern of closed convective cells, with a main flow from the heater to the lateral cold walls (in the $x$-direction) and ascending between two bright lines over the heater and descending on the bright lines (in the $y$-direction).

For small $d$, the pattern is almost two-dimensional (2D), in the form of an hexagonal pattern oriented with one of the wavevectors parallel to the heater. This pattern appears because the temperature profile at the bottom is nearly flat for small depths, while for higher depths the profile in the $x$-direction reach a maximum on the heater, and minima over the lateral walls, taking the shape of a Gaussian-like profile. The behavior of this pattern has not been studied.

When the temperature is further increased, and it approaches the threshold of the secondary instability, some drifting phase pulses can be observed (Fig. 4). These pulses are very well-defined in space, and inside them the wavenumber is smaller and about $0.8 k_{0}$, being $k_{0}$ the wavenumber of the neighborhood. The group velocity (see Fig. 5) of these drifting pulses is negative, as can be verified in Fig. 4 where the pulse propagates in opposite direction of the phase velocity. This behavior is not related to a possible hysteresis mechanism. In Section 4 , we will focus on the origin of these drifting waves.

\subsection{Time-dependent patterns}

Beyond a second threshold $\Delta T_{v}^{2}$, the stationary patterns destabilize and a second mode with a wavelength twice the stationary one $k_{1}=k_{0} / 2$ and a finite frequency $\omega_{0}$ at threshold is created. Within the experimental resolution, the bifurcation is supercritical, and no hysteresis has been found. Depending on the experimental parameters, various patterns are observed.

For small depths $d<4 \mathrm{~mm}$, the bright lines of the original pattern oscillate in opposition of phase 


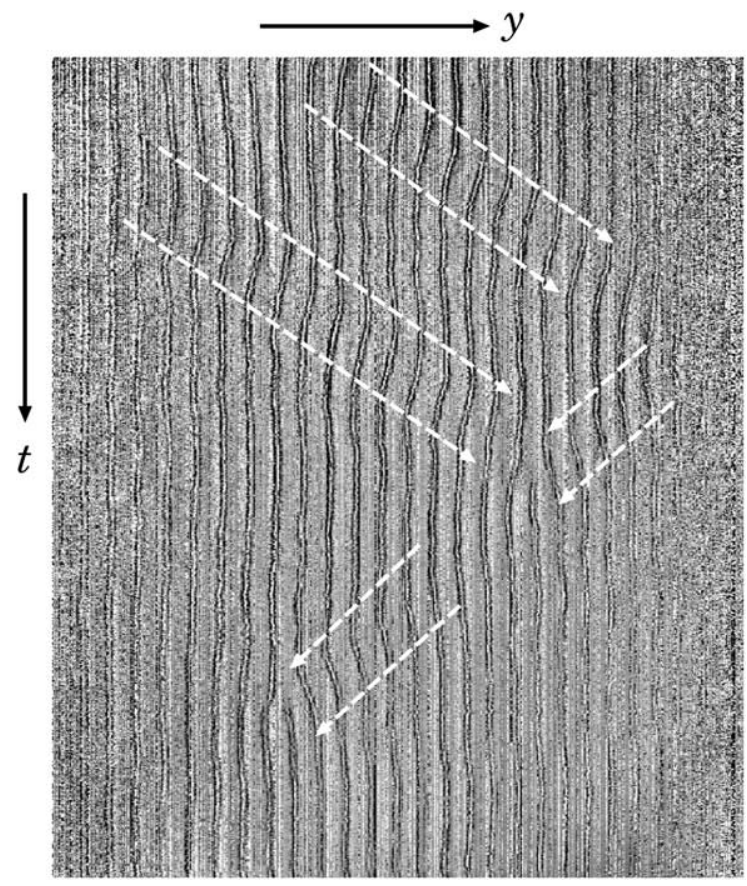

Fig. 4. Localized drifting waves. The parameter values $d=3.00 \mathrm{~mm}$ and $\Delta T_{v}=20 \mathrm{~K}$ correspond to the zone II (stationary pattern) but near the secondary bifurcation. Each pair of white dashed lines cover one propagative pulse. The arrows indicate the drifting direction (i.e. the group velocity).

(optical mode, region IIIa in Fig. 2 and spatio-temporal diagram in Fig. 6a), although for higher depths $d>$ $4 \mathrm{~mm}$, a left or right traveling wave appears (region IIIb in Fig. 2, spatio-temporal diagram in Fig. 6b).
The transition between these regions is smooth, and a coexistence of both patterns can be observed for intermediate depths ( $d \simeq 4 \mathrm{~mm}$, Fig. 6c).

The oscillatory pattern is not very stable. In fact, its spatio-temporal diagrams are plenty of defects, and the local frequency and wave number of the pattern fluctuates more than $10 \%$.

For $d>4 \mathrm{~mm}$, the oscillation disappears, and only a traveling wave with a wavelength twice the basic one and the same frequency of the oscillating pattern remains (see Fig. 6b). This pattern is very stable, and small perturbations do not affect it. A strong perturbation (the surface was tapped with an stick) switch the observed pattern to the symmetric case: from left to right traveling wave (see Fig. 7). A time-averaged profile on the reference frame of the traveling wave is plotted in Fig. 8. This profile breaks the $y \leftrightarrow-y$ symmetry.

For $d$ intermediate, the observed pattern is very complex, and there is no clear borderline between zones IIIa and IIIb. In the transitional depth, domains in the $y$-axis can be distinguished, where the pattern is either propagative or oscillating.

These two patterns have three basic modes in Fourier space. The basic stationary pattern, with wavenumber and frequency $(k, \omega)=\left(k_{0}, 0\right)$, and two traveling waves $\left( \pm k_{0} / 2, \omega_{0}\right)$. Nonlinear combination of these three modes can also appear.

Depending on the relative amplitude of each one of these modes, we will find different behaviors. When

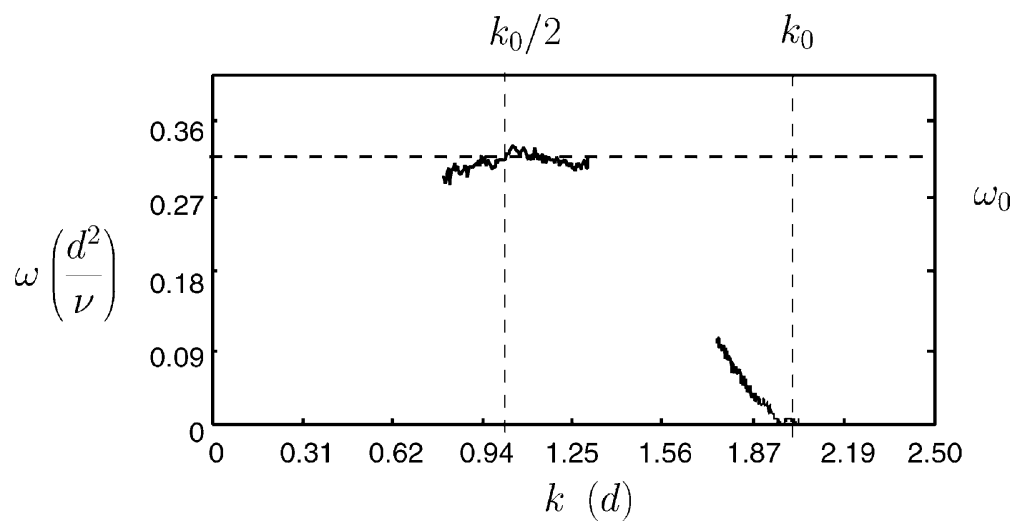

Fig. 5. Dispersion relation $\omega\left(k_{y}\right)$. Two different cases are presented: traveling waves (TW) near the point $\left(\omega, k_{y}\right)=\left(\omega_{0}, k_{0} / 2\right)$ where the group velocity is $v_{\mathrm{g}} \simeq 0$ and the stationary pattern $\left(\omega, k_{y}\right)=\left(0, k_{0}\right)$ where the $v_{\mathrm{g}}<0$. 

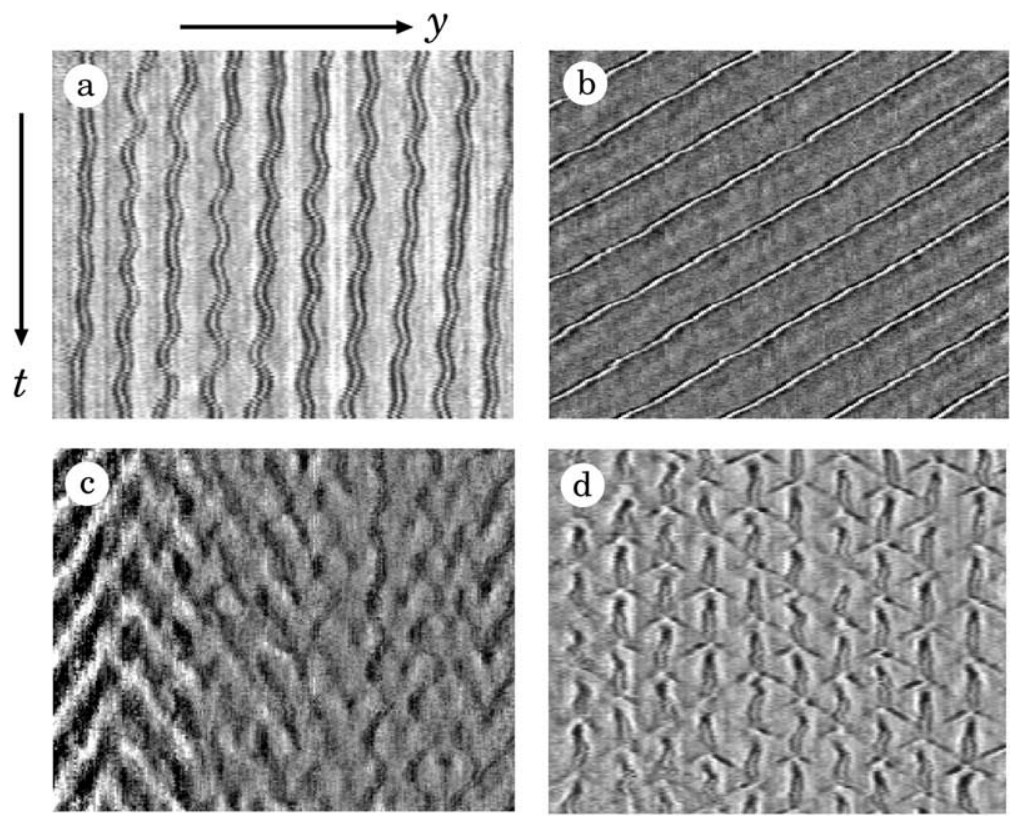

Fig. 6. Spatio-temporal diagrams of the observed patterns: (a) oscillatory pattern corresponding to $d=3.5 \mathrm{~mm}$ and $\Delta T_{v}=25 \mathrm{~K}$ (region IIIa). (b) Traveling waves corresponding to $d=4.5 \mathrm{~mm}$ and $\Delta T_{v}=15 \mathrm{~K}$ (region IIIb). (c) Intermediate pattern observed between regions IIIa and $\mathrm{b}(d=4.00 \mathrm{~mm}$ and $\Delta T=20 \mathrm{~K})$. (d) Alternating pattern corresponding to $d=4.5 \mathrm{~mm}$ and $\Delta T_{v}=25 \mathrm{~K}$ (region IIIc). The horizontal direction corresponds to the $y$-axis and time increases downwards.

the amplitudes of the stationary mode $\left(A_{\mathrm{S}}\right)$ is bigger than the amplitudes of the left $\left(A_{\mathrm{L}}\right)$ and right $\left(A_{\mathrm{R}}\right)$ traveling modes an oscillatory pattern is observed. Typically, $A_{\mathrm{S}} \simeq 6 A_{\mathrm{L}, \mathrm{R}}$ for the space-time plot shown in Fig. 6a. In the case of the traveling waves, only one of the propagating modes has a non-zero amplitude (Fig. 9).

If $\Delta T$ is further increased, a new standing pattern with a pulzation of the basic structure with a period $T$ (region IIIc in Fig. 2 and spatio-temporal diagram in Fig. 6d) arises. Pairs of contiguous bright lines appear and disappear alternatively, giving rise to a $2 \lambda_{0}$ pattern with a frequency $\omega_{0}=2 \pi / T$.

There are no sudden changes on the frequency and on the involved wavenumbers while the threshold is crossed. They changes smoothly without noticing that a new patterns exists. In fact, there is not a new instability. The only difference with the previous states IIIa and IIIb, is in the relative amplitude of the basic modes described before. $A_{\mathrm{L}, \mathrm{R}} \simeq A_{\mathrm{S}}$ at the boundary of zone IIIc, and $A_{\mathrm{L}, \mathrm{R}}>A_{\mathrm{S}}$ when we are far from the threshold.
This pattern is so stable that even very strong perturbations disappear after a transient time. Nevertheless, the amplitude of the active modes evolve in space and time.

\subsection{Characteristics of the modes}

Demodulation techniques allow to determine the characteristics of the observed patterns: the wavelengths, frequencies, phase and group velocities and the amplitudes when possible have been obtained as explained in Ref. [8].

In Fig. 10 we present the adimensional wavenumbers (characteristical scale: $d$ ) of the modes in the various observed patterns. For each pattern, we only present the wavenumber of the corresponding stationary mode. As can be seen in this figure, the wave number remains between 1.75 and 2 , and there is not a clear trend in the dependence on the temperature difference. There is no difference between the two cells (SC and BC) and the effect of changing $\Delta T_{v}$ and $\Delta T_{h}$ is not noticeable. 


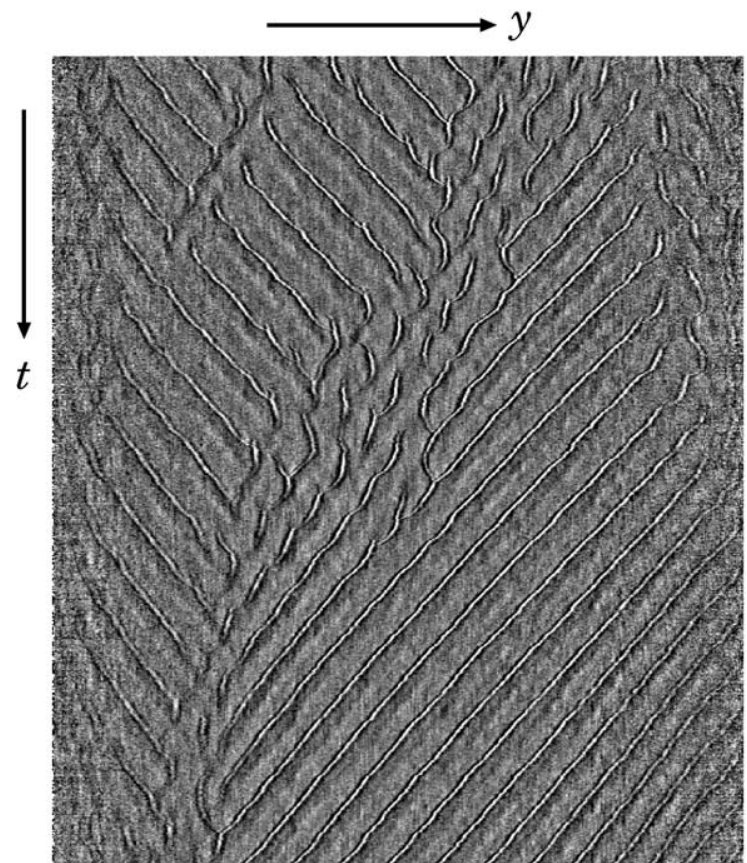

Fig. 7. Stability of a traveling wave: a right traveling wave in region IIIb is destabilized by means of a strong perturbation on the surface. This perturbation propagates and changes the wave to a left traveling wave. Time increases downwards.

On the contrary, the frequencies (characteristic time: $d^{2} / v$ ) depend strongly on the values of the parameters in the experimental cells (see Fig. 11). In the short cell (Fig. 11, top), for a given $d$ the frequency increases with the control parameter $\Delta T_{v}$. In this cell an increase of $\Delta T_{v}$ means that both horizontal and vertical gradients increase. Note that the lateral wall temperature is not controlled. For simplicity, we have plotted the frequency vs. the reduced parameter $\epsilon=$ $\left(\Delta T_{v}-\Delta T_{v}^{\text {th }}(d)\right) / \Delta T_{v}^{\text {th }}(d)$, where $\Delta T_{v}^{\text {th }}(d)$ is the threshold value of the secondary instability.

On the long cell, the horizontal and vertical gradients can be varied independently. The frequency depends strongly on both parameters. On the bottom part of Fig. 11, we present the influence of the horizontal (left) and vertical (right) temperature gradients on the frequency. Note that in this case we have not plotted the reduced parameter, because the threshold value depends on three parameters, and then, for a given $d$, there are various pairs $\left(\Delta T_{v}, \Delta T_{h}\right)$ that determine the threshold (see Fig. 2).

When we increase the horizontal gradient, the frequency increases linearly, as it happens in the short cell. This fact suggest that the secondary instability depends strongly on the basic flow, because the last one is accelerated by the bigger lateral gradient, and then the characteristic circulation times decrease. On the other hand, an increase of the vertical gradient reduces the frequency of oscillation. We cannot provide an explanation to this fact.

We have determined the group velocity $v_{\mathrm{g}}=\partial \omega / \partial k$ using the techniques described in Ref. [8] (see Fig. 5).

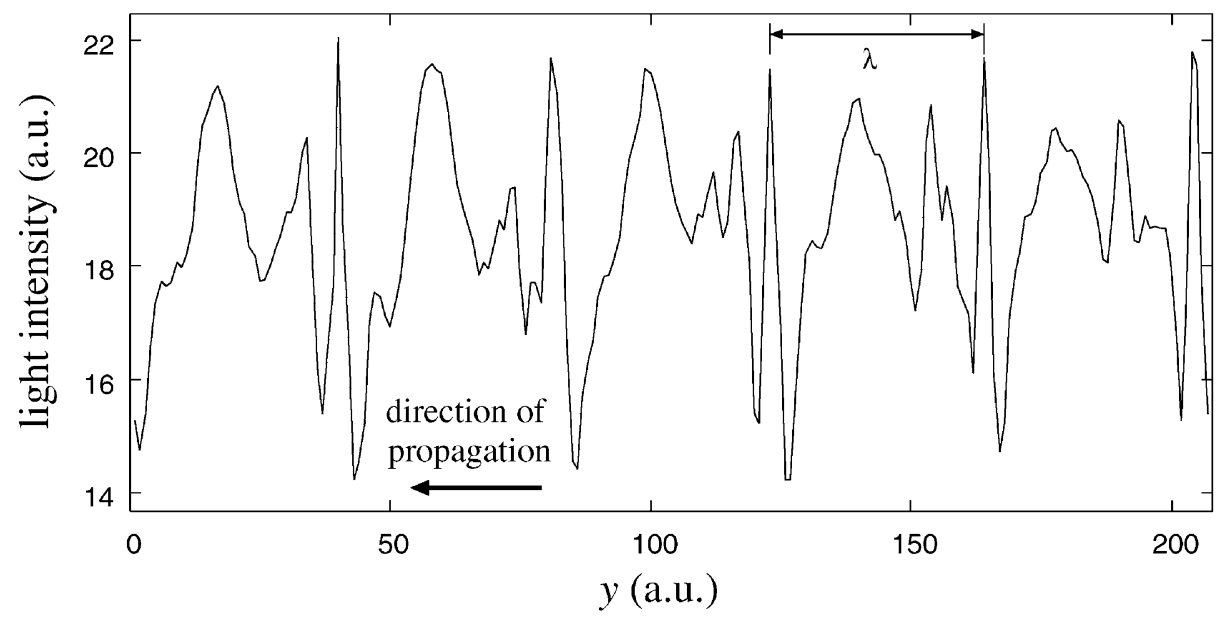

Fig. 8. Parity breaking symmetry. Intensity profile of the travelling wave averaged in the reference frame of the phase velocity. This plot covers five wave lengths of the spatio-temporal diagram shown in Fig. 6 b. 
(a)

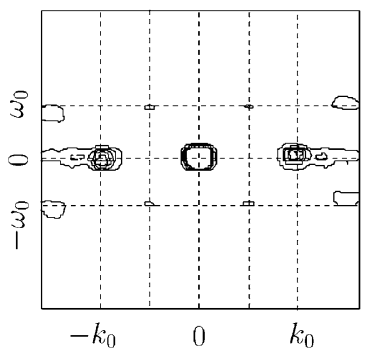

(b)

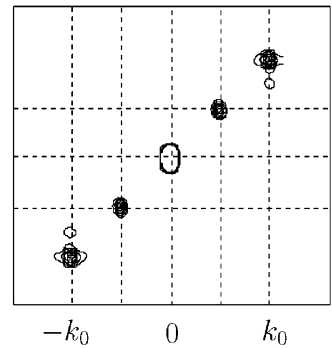

(c)

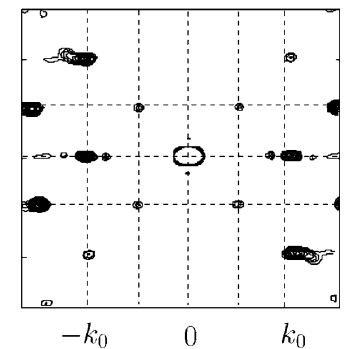

Fig. 9. Fourier spectra contour plots of the spatio-temporal diagrams presented in Fig. 6. (a) Oscillatory pattern (region IIIa), relative amplitudes: $A_{\mathrm{R}, \mathrm{L}}\left( \pm k_{0} / 2, \omega_{0}\right) \simeq 0.05 A_{\mathrm{S}}\left(k_{0}, 0\right)$. (b) Traveling waves (region IIIb). (c) Alternating pattern (region IIIc), relative amplitudes: $A_{\mathrm{R}, \mathrm{L}}\left( \pm k_{0} / 2, \omega_{0}\right) \simeq 0.5 A_{\mathrm{S}}\left(k_{0}, 0\right)$. Spatial (resp. temporal) frequencies are in the horizontal (resp. vertical) axis.
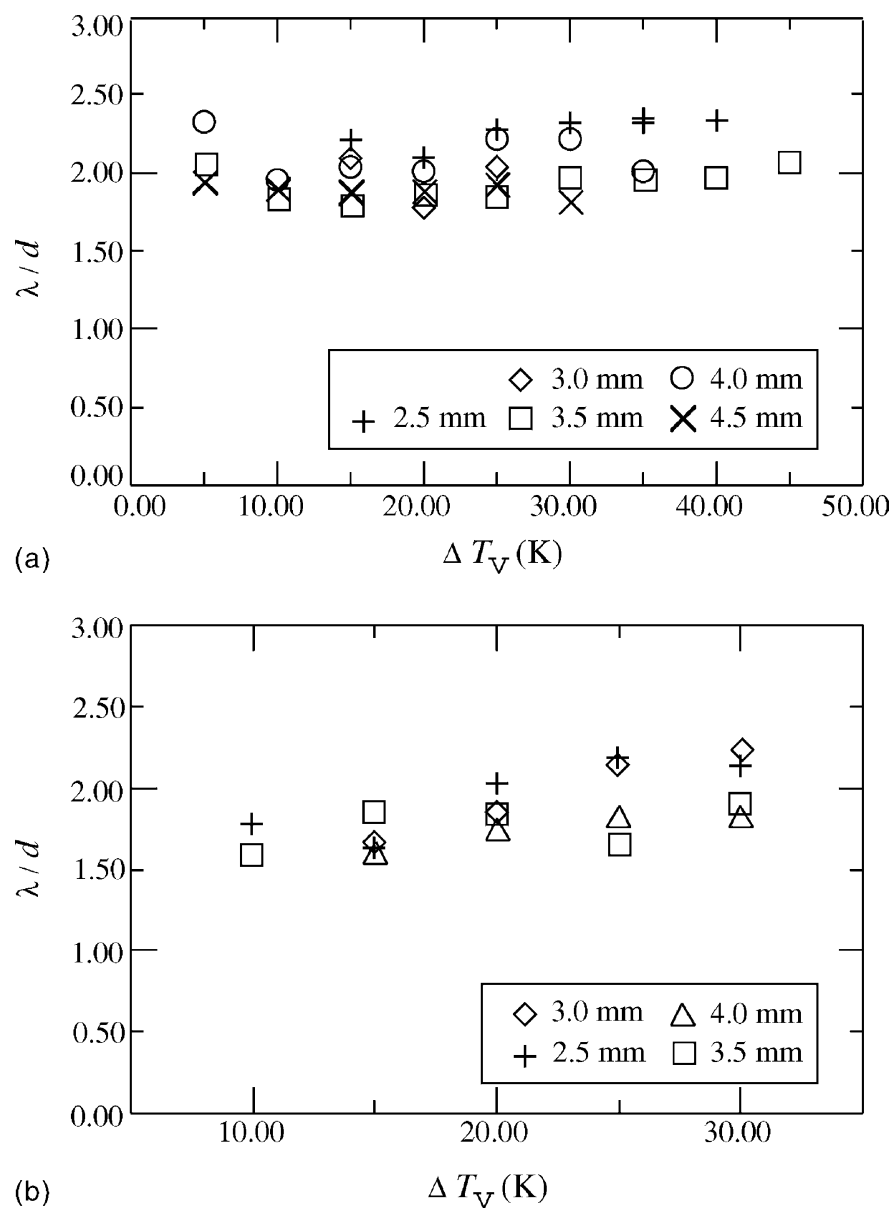

Fig. 10. Adimensional wavelength vs. depth in zones IIa and III. Top: short cell; bottom: long cell. 

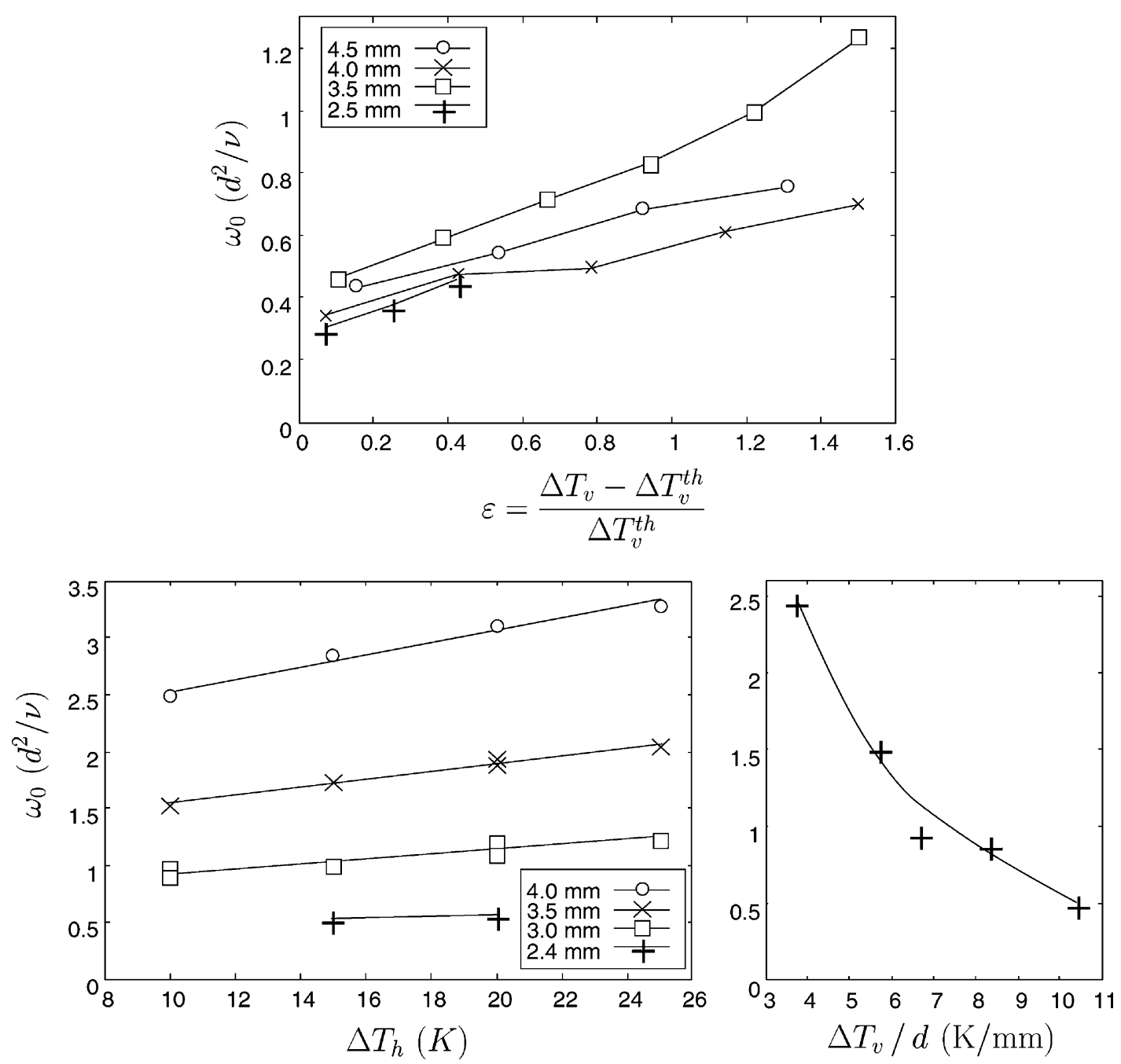

Fig. 11. Frequency of the pattern vs. depth in zone III. Top: short cell; bottom: long cell.

The phase velocity for each mode $v_{\phi}=\omega / k$ has been obtained from Fourier space. Two regimes can be clearly distinguished. For a stationary pattern, near the secondary threshold, the perturbation (see Fig. 4) propagates in opposite direction to the phase velocity. In this case we have a negative velocity group ( $v_{\mathrm{g}} \simeq$ $\left.-3 v_{\phi}\right)$.

For the travelling wave, the conclusion is very different. The group velocity is null, although the phase velocity is not zero. This implies that a small perturbation produced at a given location does not propagate, a fact that has been verified experimentally.
To ascertain what kind of secondary bifurcation takes place, the value of the amplitude of the mode as a function of the distance to threshold must be determined. Nevertheless, because of the nonlinear coupling among the various modes, a classical demodulation is not sufficient.

\subsection{Temperature behavior}

The time-dependent temperature field behavior in zone III has been studied. In all three regions ( $\mathrm{a}, \mathrm{b}$ and c) appreciable temperature oscillations are obtained. 

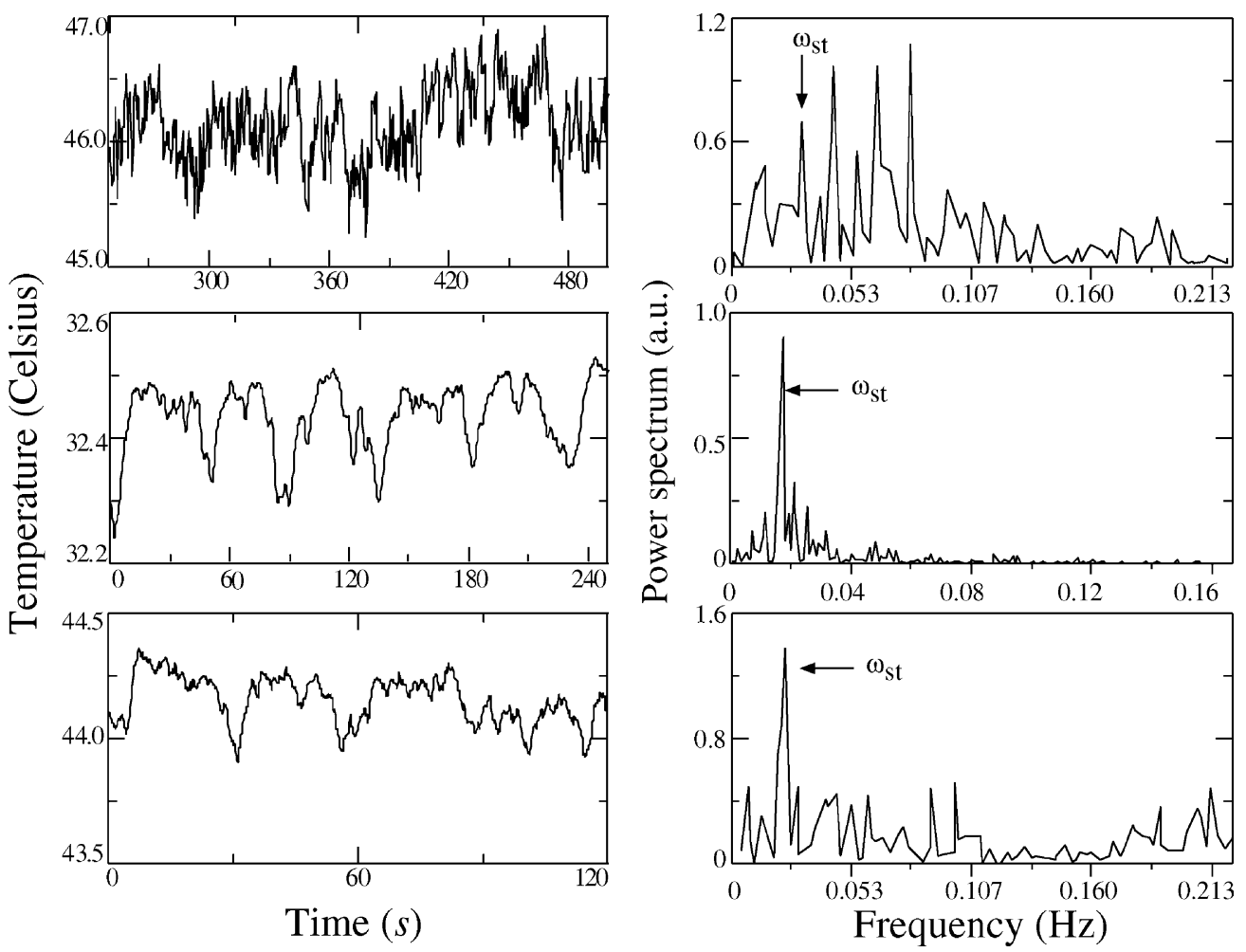

Fig. 12. Temperatures (left) and power spectra (right) for the cases (top to bottom) oscillatory (region IIIa), traveling waves (region IIIb) and alternated (region IIIc).

As shown in Fig. 12, typical oscillation amplitudes range from 0.1 to $1 \mathrm{~K}$, depending on the regime. For both alternating and traveling waves zones these oscillations have a very well-defined frequency.

On the other hand, in region IIIa (oscillatory) the thermal oscillations are irregular but with a predominant period which can be easily spotted using Fourier transforms, the biggest temperature oscillations appearing in this late case. Different harmonics and subharmonics of the pattern frequency arise in the dynamics, producing the temporal signal plotted in the upper part of Fig. 12.

Measuring the laser beam deflection, the oscillations of the temperature gradients along the two horizontal spatial directions ( $x$ and $y$ ) have been recorded. The oscillation of the deflected beam is in phase with the temperature field, and confirm that the thermal gradients in the bulk of the fluid oscillate. Moreover, the gradients in the $x$-direction (across the heater) are typically three of four times bigger than those in the $y$-direction (along the heater). Comparison between the temperature measurements, the deflected beam oscillation and the spatio-temporal diagrams allows to conclude that the bright lines in the patterns correspond to the cold regions of the flow.

The physical mechanism underlying the secondary instability has been investigated through the system response to a sudden increase of $\Delta T_{h, v}$ in the big cell. The starting point is a stationary pattern for $d=5 \mathrm{~mm}$. Suddenly, as fast as it is allowed by the experimental setup, the temperature difference of the heater is increased in $5 \mathrm{~K}$. Then, the control parameters $\Delta T_{v}$ and $\Delta T_{h}$ are increased faster than the evolution of the thermal gradients inside the flow. Under these circumstances, the frequency increases sharply, but it decreases asymptotically to the equilibrium value (see Fig. 13, bottom). This behavior is due to the relaxation of the thermal gradients across 

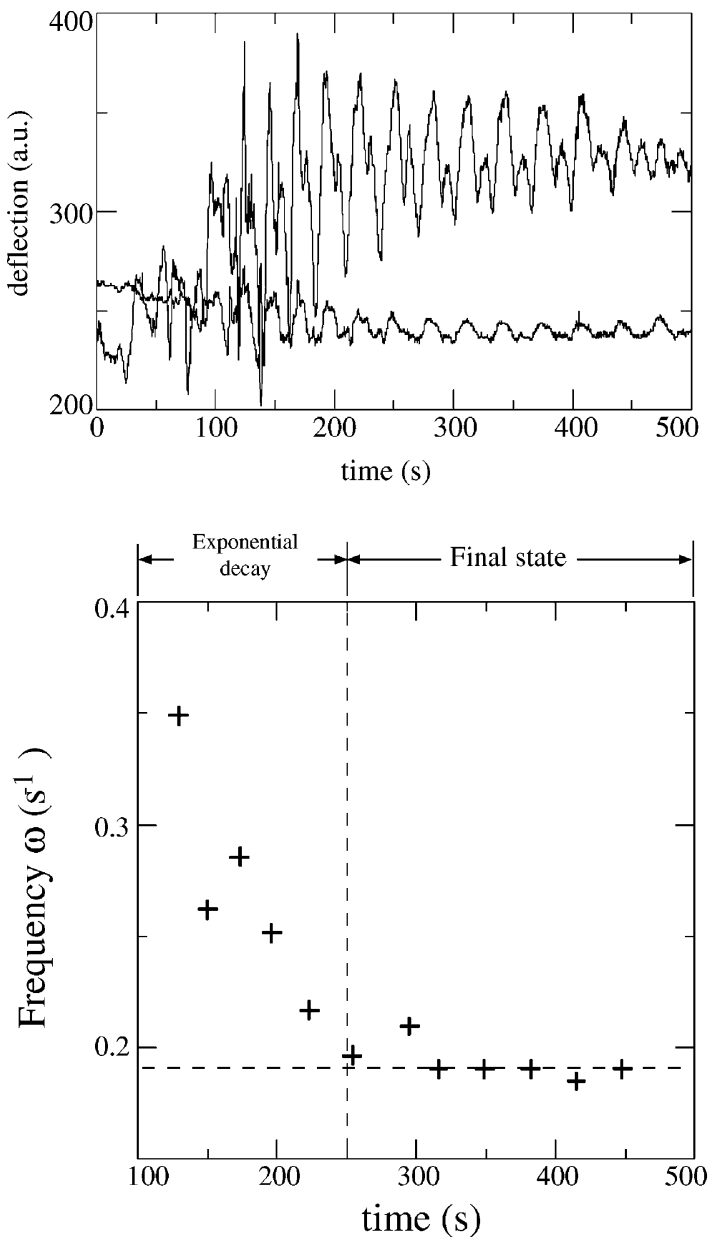

Fig. 13. Effect of a sharp increase of the heater temperature. The initial state was a one-dimensional stationary pattern and the final pattern corresponds to traveling waves. Top: deflection of a laser beam in the $x$ - (upper line) and $y$ - (lower line) direction as a function of time. Bottom: instantaneous frequency vs. time $\left(d=5 \mathrm{~mm}\right.$ and $\Delta T_{v}=15 \mathrm{~K}$.) the fluid layer, associated with the slow decaying of the laser beam deflection shown in the top of Fig. 13 (the fast oscillations with $T=\simeq 30 \mathrm{~s}$ are neglected).

Once the temperature step is applied, the local temperature gradient near the heater increases very quickly, while the gradient near the surface and the walls remains unchanged. After a transient time, these gradients decay, decreasing the local gradient near the heater and increasing the gradients near the lateral walls. As the data plotted in Fig. 13 have been obtained close $(\simeq 2 \mathrm{~mm})$ to the heater, the observed gradient decay corresponds to the decreasing gradients near the heater. Note that bigger oscillation amplitudes mean bigger local gradients.

We think that this oscillation is due to the destabilization of the thermal boundary layer growing at the bottom plate near the heater. For a fluid of $5 \mathrm{cSt}$, the thermal boundary layer has $0.5 \mathrm{~mm}$ width. When the gradient across this layer is big enough to become unstable vs. convective motions, an oscillation would appear, as a way to relax the local temperature gradient. This mechanism has been studied in a similar experimental system [40] and the control possibilities are discussed in [41].

One can ask about the role of the end walls in the dynamics. The aspect ratio in the $y$-direction is large enough to assume that these walls do not affect the dynamics. Typically $\Gamma_{y}=l_{y} / d>50$ for the short cell and $\Gamma_{y}>120$ for the long cell. Nevertheless, we have performed a series of experiments to see the effect of the thermal boundary condition on these walls. In Fig. 14 we present the effect of a normal boundary condition (center), and of an end wall warmer (left)

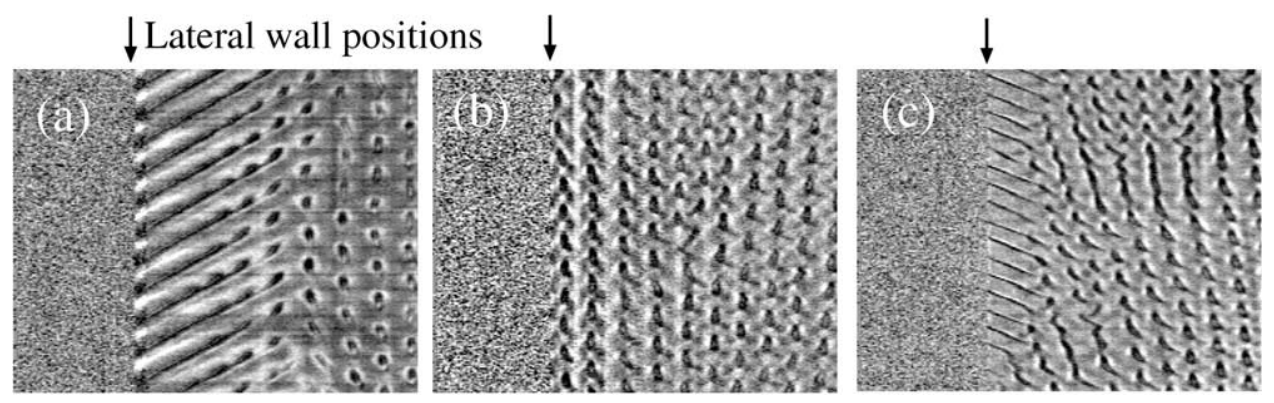

Fig. 14. Effect of the end wall thermal condition. Spatio-temporal diagrams of the dynamics when the wall is (a) colder, (b) at the same (in average) temperature, and (c) warmer than the fluid layer. The end wall is placed on the left, and time increases downwards. 
and colder (right) than the fluid. From these figures we can see that thermal gradients along the $y$-axis modify the stability of active modes. This affects the alternating (IIIc) and oscillating (IIIa) patterns. This effect is important only a few wavelengths away from the end wall, where the thermal gradients are noticeable.

This has nothing to do with the origin of the traveling waves for high $d$. The selection of the sense of propagation seems unrelated to gradients along the $y$-direction: a strong perturbation at the surface changes the pattern from right to left traveling wave (see Fig. 7).

\section{Discussion}

\subsection{Drifting phase pulses}

As it has been shown in the previous section, the dynamics of this experimental system is very rich. Let us first consider the transition to traveling waves, as it involves two modes only, i.e. the transition from the stationary pattern to the travelling wave. As plotted in Fig. 8, we have a parity breaking symmetry bifurcation. The new mode has a wave length (resp. wave number) that is twice (resp. one half) the stationary one. A model for this experiment must take into account these facts.

As introduced in Section 1, in 1990 Coullet and Iooss [29] made a first classification of the various kinds of secondary bifurcations that can appear over an stationary cellular pattern. In 1991, Goldstein et al. [30] presented an extension of this model to describe the behavior of patterns with broken space-time symmetry in hydrodynamics and interfacial phenomena.

Considering the ratio between the wavenumbers of the stationary and time-dependent patterns, the model that could describe this experiment at the threshold of the secondary instability couples the amplitude of the new mode $A$ and the phase $\phi$ through:

$\partial_{t} A=\left(\epsilon+\xi_{1} \partial_{x} \phi\right) A+\xi_{2} \partial_{x x} A-c_{1}|A|^{2} A$,

$\partial_{t} \phi=\partial_{x}\left(|A|^{2}\right)+c_{2}\left(\bar{A} \partial_{x} A-A \partial_{x} \bar{A}\right)+\partial_{x x} \phi$,

where the constants $c_{1}, c_{2}, \xi_{1}$ and $\xi_{2}$ and the parameter $\epsilon$ are complex.
This model can reproduce behaviors similar to the one plotted in Fig. 4, but just as transient. A phase modulation of the stationary pattern, coming, for example, from a local inhomogeneity in the temperature gradients, gives rise to $\partial_{x} \phi>0$. Thus, even for negative values of the real part of $\epsilon$, the amplitude of the new mode $A$ grows if the phase modulation is strong enough. A renormalization in the parameter can be introduced, $\epsilon_{n}(\phi)=\epsilon+\xi_{1} \partial_{x} \phi$, that can be locally positive. The new mode can grow in an isolated spatial region that propagates inside an stationary pattern. This could explain the drift of phase pulses observed in the fluid below the threshold of a parity breaking symmetry bifurcation. This kind of behavior has been observed in other experiments, as for example in the Rayleigh-Taylor instability [24-28], where large drifting pulses has been observed.

Nevertheless, these drifting islands are isolated domains of the propagating mode, with the appropriate wavenumber $k_{0} / 2$ and frequency $\omega_{0}$. Measuring with demodulation techniques the hydrodynamics of the pattern presented in Fig. 4, we obtain that the minimum wavenumber in the phase pulse is about $0.8 k_{0}$, far from $k_{0} / 2$, and the observed frequency is too small related to the frequency $\omega_{0}$ of the TW when the threshold has been crossed.

\subsection{Secondary instability}

Recently, Gil [31,32] has introduced an extension to these theories. There a model involving three modes is proposed, which dynamics reproduces qualitatively previous experimental results. To verify if this experiment can be described by this model, a qualitative comparison is necessary between the simulations and the experimental data.

Let us consider what happens in the region IIIa. To the experimental precision, the frequency is finite at threshold and the amplitude is zero. When the optical mode is near the threshold, and the amplitudes are small, it can be described by the destabilization of the phase of the stationary pattern:

$$
\begin{aligned}
\phi= & k_{0} x+A_{\mathrm{R}} \cos \left(\frac{1}{2} k_{0} x-\omega_{0} t\right) \\
& +A_{\mathrm{L}} \cos \left(-\frac{1}{2} k_{0} x-\omega_{0} t\right),
\end{aligned}
$$


where $\phi$ is the phase of the original cellular pattern, $k_{0}$ is the wave number of the basic state, and $\omega_{0}$ is the frequency of the pattern, finite at threshold. Finally, $A_{\mathrm{L}}$ $\left(A_{\mathrm{R}}\right)$ is the amplitude of the phase wave propagating to the left (right) along the $y$-axis.

Assuming that the basic state is $S(x, t)=$ $A_{\mathrm{S}} \exp [\mathrm{i}(\phi(x))]$ we can ask how the Fourier space of such a pattern will look like. A Taylor expansion of the term $\exp [\mathrm{i} \phi]$ gives:

$$
\begin{aligned}
\exp [\mathrm{i} \phi]= & \exp \left(\mathrm{i} k_{0} x\right)\left[\left(1+A_{\mathrm{R}} A_{\mathrm{L}}+\bar{A}_{\mathrm{R}} \bar{A}_{\mathrm{L}}+\text { h.o.t. }\right)\right. \\
& \times M(0,0)+\mathrm{i}\left(A_{\mathrm{R}}+\frac{1}{2} A_{\mathrm{R}}^{2} A_{\mathrm{L}}+A_{\mathrm{R}} \bar{A}_{\mathrm{R}} \bar{A}_{\mathrm{L}}\right. \\
& + \text { h.o.t. }) M\left(\frac{1}{2} k_{0}, \omega_{0}\right)+\mathrm{i}\left(A_{\mathrm{L}}+\frac{1}{2} A_{\mathrm{L}}^{2} A_{\mathrm{R}}\right. \\
& \left.+A_{\mathrm{L}} \bar{A}_{\mathrm{R}} \bar{A}_{\mathrm{L}}+\text { h.o.t. }\right) M\left(-\frac{1}{2} k_{0}, \omega_{0}\right) \\
& +\left(A_{\mathrm{R}} \bar{A}_{\mathrm{L}}+\text { h.o.t. }\right) M\left(0,2 \omega_{0}\right) \\
& \left.+\left(\left|A_{\mathrm{R}}\right|^{2}+\text { h.o.t. }\right) M\left(k_{0}, 0\right)+\text { h.o.t. }+ \text { c.c. }\right],
\end{aligned}
$$

where $M\left(k_{i}, \omega_{i}\right)=\exp \left[\mathrm{i}\left(k_{i} x-\omega_{i} t\right)\right]$ as usual.

Because of the nonlinearities, we are not able to obtain the values of $A_{\mathrm{R}}$ and $A_{\mathrm{L}}$ using classical demodulation techniques. For example, the $A_{\mathrm{R}}(x, t)$ of a traveling wave could be obtained from the mode $M\left(k_{0} / 2, \omega_{0}\right)$. But here we obtain $A_{\mathrm{R}}+(1 / 2) A_{\mathrm{R}}^{2} A_{\mathrm{L}}+$ $\cdots$. and the estimated error on the value of $A_{\mathrm{R}}$ is about $10 \%$. A precise calculation of the amplitudes is not possible, and even the determination of the law $A_{\mathrm{R}}$ vs. $\epsilon$ is not possible because of the nonlinearities.

A comparison with the models proposed in Refs. $[29,30,32]$ is very difficult, because the amplitudes cannot be easily determined. Nevertheless, a qualitative comparison can be performed. For example, the demodulation of Fig. 4 reveals that the drifting pulses correspond, in fact, to amplitude holes of the stationary pattern. Note that the minimum wavenumber of the drifting wave is $0.8 k_{0}$, and the frequency is very low. This pattern could be related to the drifting Bloch walls predicted by Gil [32] Further work is on the way to obtain quantitatively the amplitudes of the basic modes.

\section{Conclusions}

We have presented a new experimental system where the destabilization of an stationary cellular pattern has been observed. After a secondary bifurcation, various time-dependent patterns are created with a wavelength twice the basic one: traveling waves, oscillations (optical mode), and an alternating (standing) state have been observed. Thermal measurements reveal that the physical mechanism of the secondary instability is the destabilization of the thermal boundary layer on the bottom plate.

The characteristics of the involved modes have been determined, revealing that the frequency depends strongly on the control parameters. The stability of the patterns is very different: the oscillations are easily destabilized, whereas the traveling waves and the alternating pattern are very stable. These patterns can be described by means of three different modes plus nonlinear combinations of them. Because of the nonlinearities, a quantitative determination of the amplitudes of these modes vs. the control parameters has not been possible.

We have compared the experimental results to various theoretical models, and some similarities have been found. Nevertheless, the experimental error is too high to allow a quantitative comparison. Further work is on the way to reduce this error.

\section{Acknowledgements}

We wish to thank C. Pérez-García for stimulating discussions and also acknowledge financial support trough contracts PB98-0208 (Spanish Government), PIUNA (Universidad de Navarra, Spain) and HPRNCT-2000-00158 (DG XII, European Commission).

\section{References}

[1] F. Daviaud, J.M. Vince, Phys. Rev. E 48 (1994) 4432.

[2] J. Burguete, N. Mukolobwiez, F. Daviaud, N. Garnier, A. Chiffaudel, Phys. Fluids 13 (2001) 2773-2787.

[3] M. Vince, M. Dubois, Europhys. Lett. 20 (1992) 505.

[4] J.M. Fullana, P. Le Gal, M. Rossi, S. Zaleski, Physica D 102 (1997) 37-56.

[5] L. Pastur, R. Ribotta, Int. J. Bifurc. Chaos 11 (2001) XX .

[6] A. Prigent, C. Grégoire, H. Chaté, O. Dauchot, Physica D 174 (2003) 100-113.

[7] P. Bot, I. Mutabazzi, Eur. Phys. J. B 13 (2000) 141-155.

[8] J. Burguete, H. Chaté, F. Daviaud, N. Mukolobwiez, Phys. Rev. Lett. 82 (1999) 3252-3255. 
[9] J. Lega, J.M. Vince, J. Phys. I 6 (1996) 1417-1434.

[10] C.D. Andereck, S.S. Liu, H.L. Swinney, J. Fluid Mech. 164 (1986) 155.

[11] A.J. Simon, J. Bechhoeffer, A. Libchaber, Phys. Rev. Lett. 61 (1988) 2574.

[12] J.M. Flesselles, A.J. Simon, A.J. Libchaber, Adv. Phys. 40 (1991) 151.

[13] G. Faivre, J. Mergy, Phys. Rev. A 45 (1992) 7320-7329.

[14] M. Ginibre, S. Akamatsu, G. Faivre, Phys. Rev. E 56 (1997) 56.

[15] M. Georgelin, A. Pocheau, Phys. Rev. Lett. 79 (1997) 2698.

[16] M. Rabaud, S. Michalland, Y. Couder, Phys. Rev. Lett. 64 (1990) 184.

[17] S. Michalland, M. Rabaud, Physica D 61 (1992) 197.

[18] H.Z. Cummins, L. Fourtune, M. Rabaud, Phys. Rev. E 47 (1993) 1727-1738.

[19] J. Plumecoq, C. Szwaj, D. Derozier, M. Lefranc, S. Bielawski, Phys. Rev. A 107 (2001) 170-174.

[20] S. Ciliberto, P. Bigazzi, Phys. Rev. Lett. 60 (1988) 286.

[21] M. Dubois, F. Daviaud, O. Ronsin, P. Bergé, Travelling waves in pure fluids locally heated along wires, in: C. Pérez-García (Ed.), New Trends in Nonlinear Dynamics: Non-Variational Aspects, Physica D 61 (1992) 140.

[22] M. Dubois, R. Da Silva, F. Daviaud, P. Bergé, A. Petrov, Europhys. Lett. 8 (1989) 135.

[23] F. Daviaud, M. Dubois, P. Bergé, Europhys. Lett. 9 (1989) 441.

[24] M. Fermigier, L. Limat, J.E. Wesfreid, P. Boudinet, C. Quilliet, J. Fluid Mech. 236 (1992) 349-383.

[25] F. Giorgiutti, A. Bleton, L. Limat, J.E. Wesfreid, Phys. Rev. Lett. 74 (1995) 538.
[26] C. Counillon, L. Daudet, T. Podgorski, M.C. Jullien, S. Akamatsu, L. Limat, Europhys. Lett. 40 (1997) 3742.

[27] C. Counillon, L. Daudet, T. Podgorski, L. Limat, Phys. Rev. Lett. 80 (1998) 2117-2120.

[28] P. Brunet, J.M. Flesselles, L. Limat, Rencontre du Non-linénaire 2000, Onze, Paris, 2000.

[29] P. Coullet, G. Iooss, Phys. Rev. Lett. 64 (1990) 866.

[30] R.E. Goldstein, G.H. Gunaratne, L. Gil, P. Coullet, Phys. Rev. A 43 (1991) 6700-6721.

[31] L. Gil, Europhys. Lett. 48 (1999) 156.

[32] L. Gil, Physica D 147 (2000) 300.

[33] W. Kayser, J. Berg, J. Fluid Mech. 57 (1973) 739.

[34] R. Anthore, P. Flament, P. Gouesbet, M. Rhazi, M.E. Weill, Appl. Optics 21 (1982) 2.

[35] M.E. Weill, M. Rhazi, G. Gouesbet, J. Phys. 46 (1985) 1501.

[36] J. Burguete, H.L. Mancini, C. Pérez-García, Europhys. Lett. 23 (1993) 401.

[37] D. Maza, J. Burguete, H.L. Mancini, Int. J. Bifurc. Chaos 4 (1994) 1353.

[38] C. Pérez-García, J. Burguete, D. Maza, H.L. Mancini, Travelling convective patterns with a localized heating, in: J. Trhân-Thanh-Vân, P. Bergé, R. Conte, M. Dabois (Eds.), Proceedings of the Chaos Complexity Conference, Editions Frontiers, Paris, 1995, pp. 333-336.

[39] S. Ciliberto, F. Francini, F. Simonelli, Opt. Commun. 54 (1985) 381.

[40] H. Mancini, D. Maza, Phys. Rev. E 55 (1997) 2757.

[41] S. Boccaletti, D. Maza, H. Mancini, R. Genesio, F.T. Arecchi, Phys. Rev. Lett. 79 (1997) 5246. 